PROCEEDINGS OF THE

AMERICAN MATHEMATICAL SOCIETY

Volume 126, Number 5, May 1998, Pages 1547-1548

S 0002-9939(98)04673-5

\title{
ON A THEOREM BY DO CARMO AND DAJCZER
}

\author{
GUIDO HAAK
}

(Communicated by Christopher Croke)

\begin{abstract}
We give a new proof of a theorem by M.P. do Carmo and M. Da-
\end{abstract} jczer on helicoidal surfaces of constant mean curvature.

\section{INTRODUCTION}

Let $G$ be a one-parameter group of proper Euclidean motions of $\mathbb{R}^{3}$ of the form

$$
g_{t}(x, y, z)=(x \cos t+y \sin t,-x \sin t+y \cos t, z+h t), t \in \mathbb{R},
$$

i.e., $G$ is a group of helicoidal transformations with pitch $h \in \mathbb{R}$. In the degenerate case $h=0, G$ becomes a group of pure rotations. Up to an affine change of coordinates and reparametrization, all one-parameter groups of Euclidean motions are either of this form or are groups of pure translations.

In 1982 do Carmo and Dajczer [5] investigated surfaces of constant mean curvature (CMC-surfaces) which are generated from a plane curve by the action of a helicoidal group. They proved the following theorem:

Theorem 1.1. A complete immersed CMC-surface is helicoidal if and only if it is in the associated family of a Delaunay surface.

They proved this result by introducing for each helicoidal CMC-immersion the 2-parameter family of helicoidal surfaces given by Bour's Lemma [2] and evaluating the constant curvature condition for the elements of these families. This approach on one hand gives an explicit parametrization of helicoidal CMC-immersions. On the other hand, it reaches its goal, the proof of Theorem 1.1, in a fairly indirect way.

Since helicoidal surfaces still spawn interest [4], [7], we want to show in this note how Theorem 1.1 can be obtained in a much simpler way using a more recent theorem of Smyth [8] and some results from [3]. We state Smyth's theorem in the language of [3]:

Theorem 1.2. Let $\Phi: M \rightarrow \mathbb{R}^{3}, M$ a Riemann surface, be a complete conformally immersed CMC-surface admitting a one-parameter group of self-isometries. Then the simply connected cover of $M$ is the complex plane and the surface is either in the associated family of a Delaunay surface or its metric is rotationally invariant.

Received by the editors November 1, 1996.

1991 Mathematics Subject Classification. Primary 53A10.

The author was supported by Sonderforschungsbereich 288 .

(C) 1998 American Mathematical Society 
Here a self-isometry is an automorphism of the simply connected cover $\mathcal{D}$ of $M$, which preserves the metric of the universal covering immersion $\Psi: \mathcal{D} \rightarrow \mathbb{R}^{3}$ given by pulling back the immersion $\Phi$ to $\mathcal{D}$. For details see [3]. Those CMC-surfaces which have a rotationally invariant metric are now commonly called Smyth surfaces.

We also introduce the notion of a space symmetry of a CMC-immersion $\Phi: M \rightarrow$ $\mathbb{R}^{3}$. A space symmetry of $\Phi$ is a Euclidean motion in $\mathbb{R}^{3}$ which preserves the image of $\Phi$ as a set. The relation between space symmetries and self-isometries was also studied exhaustively in [3]. By [3, Lemma 2.15] the group of space symmetries of a Smyth surface is discrete.

\section{The Proof of Theorem 1.1}

Proof. For a given CMC-immersion $\Phi: M \rightarrow \mathbb{R}^{3}$ there exists (see e.g. [3, Theorem 2.2]) a conformal structure on $M$ such that $M$ becomes a Riemann surface and $\Phi$ becomes a conformal CMC-immersion. If $\Phi$ is also complete and in addition admits a one-parameter group of helicoidal space symmetries, then by [3, Prop. 2.12] and [3, Corollary 2.6], $\Phi$ admits also a one-parameter group of self-isometries. In particular it satisfies the assumptions of Theorem 1.2 above. Since a group of helicoidal Euclidean motions is never discrete, the surface cannot be a Smyth surface. It therefore has to be in the associated family of a Delaunay surface.

Conversely, by [3, Lemma 2.15] and [3, Prop. 3.4] each element of the associated family of a Delaunay surface admits a one-parameter group of space symmetries. Since the most general one-parameter group of Euclidean motions is a group of helicoidal transformations (with possibly degenerate pitch), all surfaces in the associated family of a Delaunay surface are helicoidal or rotational.

It should also be noted that in the language of integrable systems (the metric of a conformal CMC-immersion without umbilics satisfies the integrable sinh-Gordon equation), Theorem 1.1 also implies that helicoidal CMC-surfaces are of finite type (see [6] and [1]). Thus for helicoidal surfaces, apart from the parametrizations given in [5] and [7], there is Bobenko's parametrization in terms of theta functions [1].

\section{REFERENCES}

[1] A. Bobenko, All constant mean curvature tori in $R^{3}, S^{3}, H^{3}$ in terms of theta-functions, Math. Ann., 290 (1991), pp. 209-245. MR 92h:53072

[2] E. Bour, Memoire sur le deformation de surfaces, Journal de l'Ecole Polytechnique, XXXIX Cahier (1862), pp. 1-148.

[3] J. Dorfmeister And G. HAAK, On symmetries of constant mean curvature surfaces, preprint 197, KITCS and SFB288, 1996.

[4] B. Konopelchenko and I. Taimanov, Constant mean curvature surfaces via an integrable dynamical system, J. Phys. A, 29 (1996), pp. 1261-1265. MR 97b:53015

[5] M.P. Do CARmo And M. DAJCzer, Helicoidal surfaces with constant mean curvature, Tohoku Math. Journal, 34 (1982), pp. 425-435. MR 84f:53003

[6] U. Pinkall and I. Sterling, On the classification of constant mean curvature tori, Annals of Math., 130 (1989), pp. 407-451. MR 91b:53009

[7] T. SAsAi, On helicoidal surfaces with constant mean curvature, Tokyo J. Math., 19 (1996), pp. 39-50. MR 97c:53015

[8] B. Sмүтн, A generalization of a theorem of Delaunay on constant mean curvature surfaces, in Statistical thermodynamics and differential geometry of microstructured materials, Springer, Berlin, Heidelberg, New York, 1993. MR 94f:53012

Fachbereich Mathematik, TU-Berlin, D-10623 Berlin

E-mail address: haak@sfb288.math.tu-berlin.de 